\title{
A qualitative study to identify factors that influence patients' decisions to call Emergency Medical Services for syncope
}

\section{Tamara M. Williamson}

University of Calgary

\section{Mary Runte}

University of Lethbridge Faculty of Management

\section{Tigana Runte}

Libin Cardiovascular Institute of Alberta

\section{Satish Raj}

Libin Cardiovascular Institute of Alberta

\section{lan Blanchard}

\section{Alberta Health Services}

\section{Robert Sheldon}

Libin Cardiovascular Institute of Alberta

\section{Tavis Campbell ( $\nabla$ campbet@ucalgary.ca )}

University of Calgary

\section{Kathryn King-Shier}

University of Calgary Faculty of Nursing

\section{Research article}

Keywords: syncope, treat-and-refer, EMS, emergency medical services, paramedic, health care utilization, decision-making

Posted Date: May 18th, 2020

DOl: https://doi.org/10.21203/rs.3.rs-28350/v1

License: (c) (i) This work is licensed under a Creative Commons Attribution 4.0 International License. Read Full License 


\section{Abstract \\ BACKGROUND}

Emergency medical services (EMS) assessment and presentation to the emergency department (ED) for syncope (fainting) often results in hospital admission and costly diagnostic testing that rarely benefits patients. Protocols that support paramedics to assess, treat and refer low-risk syncope may allow for ED transport of only high-risk patients. The development and successful uptake of such protocols is limited by a dearth of information about factors patients consider when deciding to seek EMS care following syncope.

\section{OBJECTIVE}

We aimed to explore decision-making processes of individuals with syncope when deciding whether (or not) to call EMS after fainting as a starting point in the development of prehospital risk-stratification protocols.

\section{METHODS}

Twenty-five adults (aged 18-65 years) with a history of $\geq 1$ syncopal episode were recruited across four Canadian provinces. Individual semi-structured interviews were conducted, recorded, and transcribed. Straussian grounded theory methods were used to identify common themes and a core (overarching) category.

\section{RESULTS}

Four common themes were identified: (a) previous experiences with the healthcare system (e.g., feeling dismissed), (b) individual patient factors (e.g., age, comorbidities), (c) attitudes and beliefs (e.g., concerns about burdening the health care system, believing syncope is "not serious enough" to seek care), and (d) contextual factors (i.e., influence of important others, symptom severity). Perceived judgement of the patient by EMS was identified as the overarching core category that influenced patients' decision-making to seek care.

\section{CONCLUSION}

The decision to seek EMS care after syncope is a complex process involving patient consideration of past experiences, pre-existing attitudes and beliefs, and individual- and contextual factors. Patients' previous experiences of judgement and dismissal by EMS for fainting may interfere with patient receptiveness to traditional EMS protocols for syncope. These barriers could be targets for training and education for 
paramedics and help to inform the development of prehospital protocols to improve care and satisfaction among patients with syncope.

\section{Introduction}

Syncope (fainting) presentations to emergency departments (ED) in Canada comprise $1-3 \%$ of annual ED visits $(1)$. One large $(N=3,662)$ prospective study revealed $66 \%$ of patients with syncope were transported to the ED by ambulance(2). Whereas nearly two-thirds of ED visits for syncope result in lowrisk diagnoses (e.g., vasovagal syncope, orthostatic hypotension)(1), 10-40\% of patients are admitted to hospital and/or subjected to time-consuming, costly diagnostic assessment that rarely ( $<1 \%$ of patients) improves outcomes(3). Thus, the current standard of care for syncope has been described as inefficient, inconsistent, and may be inconvenient and/or distressing to patients $(2,3)$.

Limitations in current approaches to emergency syncope management highlight a need for riskstratification procedures for emergency medical services (EMS) to identify patients at low-risk of adverse outcomes and refer them to alternative, patient-centered services (e.g., interdisciplinary specialty units, outpatient clinics) $(2,4,5)$. EMS protocols have been developed and successfully implemented for other low-risk conditions (hypoglycemia, supra-ventricular tachycardia) that support paramedics to assess, treat, and refer patients to alternative care pathways, including leaving the patient at home $(6,7)$. Yet, qualitative interviews with paramedics suggest patients may be resistant to complying with treat-andrefer protocols by refusing, for example, to be referred to non-emergency alternatives, or discharged onsite(8-10). Further, little is known about why some patients with syncope do not contact EMS and/or present to the ED after they faint.

Elucidating the factors that individuals with syncope consider in determining whether (or not) to access EMS may be an important first-step to ensuring a risk stratification protocol will meet patients' needs and expectations $(11,12)$. Ultimately, using a patient-centered approach to developing prehospital syncope protocols has the potential to promote better protocol uptake, increased patient satisfaction, and more optimal patient outcomes.

\section{Objective}

We aimed to investigate decision-making processes of non-senior adults (18-65 years) with syncope when deciding whether (or not) to call EMS for themselves, or others, after fainting. This study represented an initial exploration into patients' perspectives on EMS utilization for low-risk syncope to help inform subsequent development of prehospital fainting protocols in the Community Alternatives to Syncope Management to the Emergency Room (CASMER) project.

\section{Methods}


Straussian grounded theory (13) was used to examine and characterize patients' decision-making about accessing EMS for syncope. Grounded theory has been described as an effective methodology for constructing comprehensive theory about human processes and behavior(13). Given the dearth of research on the emergency healthcare utilization patterns of patients with syncope, this method was chosen to provide a theoretical 'starting point' to help inform future treatment protocols(12).

\section{Patient and Public Involvement}

Patient partners were involved at all stages of this study, including developing the research question, study design, data collection, data analysis, and dissemination of the findings. The patient co-lead (MR), an experienced qualitative researcher with extensive interviewing experience, developed study materials (e.g., protocols, interview guides), conducted interviews, trained and supervised the research assistant (TR, also a patient partner), and co-led the data analysis process. MR and TR were also involved in knowledge translation via preparation of the present manuscript and presentations of parts of this work to patients, the public, and the academic community.

\section{Participants and Sampling}

Purposive sampling was utilized to obtain a sample representing a range of individuals with diverse experiences of EMS and ED utilization for syncope(13). Snowball sampling (14) was initiated by MR and the principal investigator (SR). As recruitment progressed, referrals to potentially eligible participants were also received from study participants. Given the nature of the sampling procedures, some participants had pre-existing relationships with the interviewers (e.g., friends, colleagues, or acquaintances with syncope). Participants were contacted by phone and invited to participate if they were: (a) 18-65 years old, (b) living in Canada, and (c) had fainted $\geq 1$ time at any point in their lives. Seniors ( $>65$ years) were not sampled for the present study given that older individuals may be less likely to present with syncope that is "low risk", owing to age-related comorbidities. Participants may, or may not have, sought EMS care following their fainting episode - we were interested in decision-making processes that fainters engaged in regarding contacting EMS, regardless of whether EMS was eventually called. No participants who were approached refused to participate or dropped out after consenting into the study. Sampling continued until theoretical saturation was reached, meaning no new themes emerged from the data with continued sampling $(13,15)$.

\section{Ethics Approval and Consent.}

This study was approved by the University of Calgary, Conjoint Health Research Ethics Board, and each participant provided individual informed consent. 


\section{Data Collection}

Participants completed a demographic questionnaire and engaged in one-on-one semi-structured interviews (in-person or over the phone, according to preference; no repeat interviews were conducted). The interviews were conducted by MR and/or TR, and audio-recorded. The interviewers both identify as women. TR received extensive training and was supervised by MR throughout data collection. Interviews were audio-recorded and ranged in duration from 10-90 minutes. Participants were initially invited to describe their experience/history with fainting, including their experience with EMS if applicable. Next, the interviewer inquired about participants' perspectives regarding EMS utilization using questions, such as: 1) Did you, or a bystander, call EMS after you fainted?; 2) Did you want EMS to be called? Why or why not?; and 3) What factors would you consider in deciding whether or not to call EMS for yourself, or for another person, who fainted? Follow-up questions and probes were directed as-needed to elicit additional detail about their experiences.

As data collection progressed, interview questions were continually refined to reflect new insights provided by the participants $(13,15)$.

\section{Data Analysis}

Demographic data were entered into IBM SPSS Statistics 25 and descriptive analyses (means, $S D$, range, proportions) calculated. Audio-recordings of interviews were transcribed verbatim by a third-party transcription service. NVivo 12 Plus software was used to organize the qualitative data. First, interview transcripts were read and common elements/themes coded using a descriptive label. Second, axial coding was conducted, and broad categories were named. Third, relationships between the categories were examined to identify the core category. This core category guided the construction of a comprehensive theory about patients' EMS decision-making processes. Regular team meetings were held to establish consensus among team members (MR, TR, KKS, TW) and discrepancies in interpretation/coding were resolved through team discussions. Pseudonyms were assigned to enable differentiation between data provided by participants of the same age and gender.

Rigour(13) was established through several aspects of the methodology. First, the interviewers were patient partners with both knowledge and lived experience regarding EMS encounters for syncope. These individuals were therefore in an optimal position to administer insightful follow-up questions/probes and refine interview questions as-needed to bolster the credibility (i.e., extent to which the data captures true phenomena) of the data(13). Second, data accuracy was ensured by performing multiple, thorough reviews of interview transcripts by multiple members of the study team (MR, TR, KKS, TW). Several meetings scheduled over a one-year period provided opportunities to examine and re-examine potential categories and reach consensus among the team. Last, the process of data analysis was recorded in rigorous detail to enhance credibility and reproducibility of the results.

Results 
Twenty-five participants ranging in age from 18-65 years (33.5 \pm 14.9$)$ participated in the study (see Table 1). The sample was predominantly women (72\%) with greater than a high school education (88\%). Over half the sample were employed full- or part-time outside of the home, whereas $32 \%$ were students. Participants were from four Canadian provinces (British Columbia, Alberta, Ontario, and Quebec).

The participants described various factors influencing the decision-making process about whether to call EMS following a syncopal event, which were categorized into four common themes (Fig. 1, Table 2). Overall, participants reported concerns about judgement as a critical consideration in their EMS utilization decisions (Core category; Fig. 1, Table 2).

\section{Theme 1: Previous Healthcare Experiences}

Participants frequently described previous interactions with EMS that influenced their decisions to seek (or not seek) assistance after fainting. Two participants reported positive experiences with EMS, however most indicated past negative interactions were salient when considering not to seek care.

I think the main factor that affects [the decision to call EMS] is your history with medical services... If your experience is negative, you're much less likely to pursue help when you need it, which has certainly been my history, and that of my family. (Nicole, 35, woman)

\section{Feeling Dismissed}

Most participants described experiences where they interpreted their syncope symptoms as being dismissed by EMS and other medical personnel:

... I've been dismissed enough by doctors that unless something drastic has happened in relation to my medical condition, I don't want to go [to the ED] anymore. Sometimes that's at a risk to myself, but the experience of dismissal has happened so often.... (Ben, 30, man)

Nicole, a 35-year old woman, said "the doctors didn't really care, [that] was [a] big problem. They just sort of dismissed it." Jane, also 35, stated: "I have a very negative image of paramedics. When they come to pick you up, they'll just dismiss you."

Participants speculated about potential reasons for 'being dismissed', including the

common experience that their syncope symptoms often resolve prior to EMS arrival. For example, Amy (19) said "it (syncope) has to be more or less, caught in the act, for notice to be taken of it, or for you to be taken seriously by doctors." Participants reported the experience of their symptoms being downplayed, describing their treatment by EMS as 'disrespectful'.

(He) goes, 'Oh, it's just fainting. Oh, just throw a little water in her face. Oh, does somebody have an onion? Haha.' So yeah, it's not something I would go into ER for or to a medical professional for because 
sometimes they're not the nicest people. (Jane, 35, woman)

Further, several women participants endorsed beliefs that women who faint are more likely to be dismissed than men. Participants attributed this to a perceived bias among EMS/physicians that syncope is typically a symptom of benign, feminine conditions (e.g., menstruation, menopause, or pregnancy).

I think we still are stuck in that 1800 s view of a woman fainting like... It's not something serious... "Oh, she's going through menopause. Oh, she's got her period." They brush it off. (Megan, 30, woman)

\section{Theme 2: Individual Factors}

Patient-level factors such as age, medical history, and medical comorbidities were frequently provided as important practical considerations when deciding to seek medical care for oneself or others who faint.

\section{Age}

In general, participants reported that they would call an ambulance for a senior who fainted, due to the increased risk of serious medical concerns among older people.

...Older people fainting is much more serious because you break your hip, you break something... So the care and concern that is given to them, I feel, is much more than a 30 year old fainting. (Jane, 35 , woman)

In contrast, being "younger" was associated with a perception that fainting should not require EMS care.

Fainting just seems like a silly thing to complain about. At my age at least. (Kayla, 21, woman)

\section{Medical History}

Participants considered their own medical history, and fainting history in particular, when deciding whether to contact EMS. Individuals who fainted repeatedly/frequently considered fainting to be a 'normal' experience, not worthy of medical attention. For example, Holly (19, woman) indicated "I've fainted enough times that it doesn't really strike me as being a big deal." Kayla (21, woman) further explained: "I just wake up [after fainting] and dust myself off, carry on my day, like 'oh I have class in ten minutes."”

In contrast, when asked what would prompt them to call EMS if someone else fainted, nearly everyone indicated they would call if they were not familiar with that person's medical history.

\section{Patient Comorbidities}


Participants indicated that they would be inclined to call EMS for another person who fainted if they believed there was a more serious underlying medical condition.

I think certainly (I would call EMS) if they have been diagnosed with some major medical condition and if they have a medic alert bracelet saying so. (Luke, 35, man)

\section{Theme 3: Attitudes And Beliefs}

Participants frequently endorsed personal attitudes and beliefs about the use of healthcare resources, and about syncope in general, that influenced decision-making.

\section{Burdening The System}

\section{Numerous participants believed that accessing EMS was tantamount to "being a burden" on others and, in particular, the healthcare system. Participants wanted to avoid wasting hospital resources, and simply disliked the experience of 'needing help' from others.}

\section{[1] didn't want to be a burden on the medical system for something that wasn't an emergency... (Sarah, 60, woman)}

\section{Beliefs about Syncope}

Many participants expressed beliefs about fainting that seemed to influence their EMS utilization decisions. Syncope was often described as "not a big deal", and therefore not worthy of care/attention. In reference to an experience at the ED after fainting, June (37, woman) stated that "basically it was no big deal and they said I didn't need to be there... a bit embarrassing to be there for no reason". Reflecting on their fainting experiences, many participants dismissed their symptoms and suggested that calling EMS would have been (or was) "too much of a fuss" or "excessive". Curtis (28, man) indicated that he did not seek emergency medical assistance after fainting because "it was just a normal boring everyday faint thing that would be a waste of time to pursue".

Gender-specific beliefs. Several women in the sample used gendered language to describe syncope and fainting symptoms. Feminine (e.g., "girly", "damsel in distress", "swooning", and "dramatic") and infantilising (e.g., "whiny baby", and "silly"), terms emerged throughout the interviews in reference to 
fainting, often in reference to why patients felt (or would have felt) embarrassed for contacting EMS after fainting.

Oh, it just seemed like such a, I don't know, girly thing to do. (Paula, 38, woman)

\section{Theme 4: Contextual Factors}

Participants incorporated unique characteristics of the fainting event into their decision-making about calling EMS.

\section{Influential Others}

Influential others, including friends and family members and third-party bystanders, impacted decisionmaking in various ways. Participants often described events where one of these people called an ambulance while they were unconscious, or against their wishes/instruction.

When I fainted at the office, they lost their minds. They called [EMS]. I had to go to the hospital, and ...you know that you're going to be fine. (Megan, 30, woman)

Other participants indicated they sought care in order to mitigate concerns and fears expressed by their loved ones. Some participants reported seeking advice regarding how to respond to the faint and were advised by loved ones to call an ambulance.

I remember one of my girlfriends was an RN. I called her, and I was like, "What...is going on? Why am I feeling like this?" She's like, "You need to call the ambulance... You need to call 911."(Megan, 30, woman)

\section{Symptoms}

Symptoms, including injuries sustained from falling, were extremely influential in the decision to seek EMS care.

Symptom severity. Nearly every participant indicated symptoms that were "serious" and/or "abnormal" given their syncope history would prompt them to seek EMS care after fainting. These included: extended period of unconsciousness (perceived timeframes warranting medical attention ranged from $>2$ seconds to > 15 minutes of unconsciousness); vomiting; shortness of breath; heart palpitations; pain; convulsions; and blurred vision. Participants who fainted frequently and were familiar with their own typical symptoms indicated that symptoms would need to be "out of the ordinary" to justify a call to EMS. Megan, a 30-yearold woman who faints frequently, described an occasion when abnormal symptoms made her decide to call an ambulance: 
Not only did I feel super lightheaded, but there was a pain in my chest, and it felt different than all the other times. When l'd fainted [previously], it was usually pretty quick, and l'd wake up, and l'd feel fine... That's what made me call is that it was different.

Fall-related injuries. Several participants indicated that, in general, they only contact EMS if physically hurt from a faint. One participant described lying about the cause of an injury sustained during a faint, to avoid "admitting" to EMS that she had fainted:

I wanted to lie to the paramedics and the hospital staff and say that I just fell, when it was actually that I fainted and fell. So I left out that information completely because I know how they react when you tell them I fainted. (Jane, 35, woman)

Some participants described injury as a desirable justification for the use of EMS.

I know that this is going to sound weird, but I was happy that I had hurt my knee. Just getting all this attention for something normal like a faint would have been embarrassing. (Holly, 19, woman).

\section{Core Category: Judgement}

The core category underpinning participants' experiences of deciding to access EMS was the perception of judgement. There was a salient belief that EMS typically judge syncope as trivial, and negatively evaluate patients for utilizing healthcare resources unnecessarily. Past experiences of feeling 'dismissed' by 'the system' contributed to apprehension about being judged. The prospect of being judged unfavorably for calling EMS was a powerful deterrent from seeking care. For example, Amy (19, woman) described feeling "worried" about calling EMS out of concern that "I'd call, and (the symptoms) would stop, and (EMS) would get there, and...I'd be 100\% fine. And they'd be like, 'why are you wasting our time?".

\section{Discussion}

To our knowledge, this study was the first to identify factors relevant to individuals with syncope making the decision to contact EMS after fainting. The results have relevance to the development of prehospital protocols for low-risk syncope that will be acceptable to patients who faint and encourage appropriate healthcare utilization patterns.

While the 'weight' assigned to various factors during decision-making likely varies across individuals, typical conditions that deterred participants from contacting EMS included: prior negative interactions (e.g., feeling dismissed); perceptions that symptoms are minor; regained consciousness rapidly; and beliefs about "burdening" the medical system. In contrast, participants would consider calling EMS if: symptoms were severe/abnormal; they were injured; older age/serious medical comorbidities; or they 
were urged to seek care by an influential other (loved one, bystander). Overall, concerns about being judged underpinned the decision-making process, and in this sample, the number and strength of deterring factors frequently 'outweighed' factors that promoted care-seeking.

Several of the findings are consistent with previous qualitative research about emergency care-seeking decisions in other health domains. In a qualitative investigation of delay in contacting EMS for acute myocardial infarction (AMI), patients with AMI considered: (a) perceived symptom severity (e.g., pain intensity), (b) perceived risk posed by symptoms, (c) previous experience with AMI symptoms, and (d) the situational context (e.g., presence/absence, and advice of, other people)(16). Similar themes were identified in a sample of Chinese patients with AMI who delayed calling an ambulance following the onset of symptoms(17).

The results are also consistent with previous qualitative studies describing hesitancy in care-seeking due to concerns about "burdening" others and "wasting" healthcare resources $(16,18,19)$. In an exploration of EMS/ED use among individuals with epilepsy, a majority of individuals with epilepsy reported they attend the ED only when injured in the seizure, in an effort to avoid "wasting" the hospital's time(20). Of importance, this apparent aversion to being perceived as a "burden" to EMS may serve to enhance patients' attitudes towards ED-alternatives, and increase receptiveness to referrals to outpatient clinics and online resources for syncope.

Concerns about being judged and dismissed by EMS were prominent in this sample and strongly influenced decisions to avoid seeking emergency care. These concerns appeared to be driven by a few common factors, including previous invalidating healthcare interactions and attitudes/beliefs that syncope is a "trivial", condition. Among women in this study, perceptions that syncope is "girly" and/or "childish" also deterred care-seeking behaviour. Improving the quality of patient-EMS interactions postsyncope through EMS training initiatives could reduce apprehension about contacting EMS for syncope, and improve receptiveness to risk stratification protocols. Patient-provider communication training has been extensively studied among physicians, and is effective in improving patient satisfaction and compliance $(21,22)$. Past studies on communication training for EMS, however, have focussed primarily on interprofessional communication(23-25), rather than general patient-EMS interactions. There is a need for further research to develop and test interventions for increasing sensitive responding by EMS when attending to patients who have fainted. Improved EMS communication may help individuals with syncope feel validated, encourage appropriate use of EMS, and increase compliance with assess, treat, and refer protocols.

\section{Limitations}

Our sample consisted primarily of White women who were highly educated. Therefore, the proposed decision-making model may not generalize to ethnic minorities, men and individuals of lower socioeconomic status(19). Seniors $>65$ years were not interviewed for this study, as it was recognized that their decision-making about calling EMS for syncope may differ substantially from younger and middle- 
aged adults(26). Further research targeting older adults is needed to identify unique EMS utilization patterns in this vulnerable population. In addition, participant diagnoses were not recorded, so we could not determine whether participants indeed fell into low or high-risk syncope categories. Participants with high-risk diagnoses may consider and weigh factors differently in deciding to call EMS, relative to those with low-risk syncope.

The participants' experiences and perspectives suggest the traditional EMS paradigm of assess and transport to the ED for further assessment may not be acceptable to many individuals who faint.

Developing an assess, treat and refer-style protocol, similar to present procedures for hypoglycemia and SVT $(6,7)$, may be important to achieving patient satisfaction in this population. Protocols that circumvent the ED may be desirable for many patients who wish to avoid feeling like a "burden" on the healthcare system. Current barriers to seeking EMS care, including experiences of judgement/dismissal, could be targets for training and education for paramedics, emergency medicine physicians, and ED staff. Education for paramedics could include patient perspectives from this study coupled with patientprovider communication training to increase sensitive responding towards patients who faint.

\section{Declarations}

\section{Availability of Data and Materials:}

The dataset and materials from the current study is available from the corresponding author upon reasonable request.

\section{Prior Presentations:}

\section{Parts of this work were presented at the 2018 Cardiac Arrhythmia Network of Canada (CANet) Annual Scientific Conference in Ottawa, Ontario, Canada, and at the 2018 American Autonomic Society Annual Meeting, Newport Beach, CA, USA.}

\section{Competing Interests:}

The authors declare that they have no competing interests.

\section{Funding:}


This work was supported by a Cardiac Arrhythmia Network of Canada (CANet) Strategic Research Grant (Community Alternatives for Syncope Management in the Emergency Room [CASMER]; 2017-2020). Ms. Williamson was supported by a 2018 Alberta Strategy for Patient-Oriented Research (SPOR) Graduate Studentship for her contribution to this work.

\section{Authors' Contributions:}

TW assisted with data analysis (coding and interpretation of themes), wrote the manuscript, and revised the manuscript. MR co-conceptualized the study, collected, analyzed, and interpreted the data, provided expert consultation regarding qualitative research methods, and critically revised the manuscript. TR collected the data, assisted with data analysis and interpretation, and critically revised the manuscript. SR conceptualized the project, acquired funding, and critically revised the manuscript. IB consulted with the PI during project conceptualization, and critically revised the manuscript. RS co-conceptualized the project, acquired funding, and critically revised the manuscript. TC supervised the student collaborator (TW) and critically revised the manuscript. KKS provided expert consultation regarding the study design, qualitative research methods, data analysis/interpretation, and manuscript preparation. KKS also critically revised the manuscript.

\section{Acknowledgements:}

The authors wish to acknowledge all of the individuals with syncope who participated in this qualitative study.

\section{References}

1. Sandhu RK, Sheldon RS, Savu A, Kaul P. Nationwide Trends in Syncope Hospitalizations and Outcomes From 2004 to 2014. Can J Cardiol. 2017;33(4):456-62. 
2. Thiruganasambandamoorthy V, Taljaard M, Stiell IG, Sivilotti MLA, Murray H, Vaidyanathan A, et al. Emergency department management of syncope: need for standardization and improved risk stratification. Intern Emerg Med. 2015;10(5):619-27.

3. Sheldon RS, Morillo CA, Krahn AD, O'Neill B, Thiruganasambandamoorthy V, Parkash R, et al. Society position statement standardized approaches to the investigation of syncope: Canadian cardiovascular society position paper. Can J Cardiol. 2011;27(2):246-53.

4. Costantino G, Sun BC, Barbic F, Bossi I, Casazza G, Dipaola F, et al. Syncope clinical management in the emergency department: a consensus from the first international workshop on syncope risk stratification in the emergency department. Eur Heart J. 2016;37(19):1493-8.

5. Sun BC, Costantino G, Barbic F, Bossi I, Casazza G, Dipaola F, et al. Priorities for emergency department syncope research. Ann Emerg Med. 2014;64(6):649-55.e2.

6. Minhas R, Vogelaar G, Wang D, Almansoori W, Lang E, Blanchard IE, et al. A prehospital treat-andrelease protocol for supraventricular tachycardia. CJEM. 2015;17(4):395-402.

7. Alberta Health Services. EMS Medical Control Protocols. [Internet]. 2019 [cited 2019 Jul 1]. Available from: https://www.albertahealthservices.ca/info/page3165.aspx.

8. Snooks HA, Anthony R, Chatters R, Dale J, Fothergill R, Gaze S, Halter M, Humphreys I, Koniotou M, Logan P, Lyons R. Support and Assessment for Fall Emergency Referrals (SAFER) 2: a cluster randomised trial and systematic review of clinical effectiveness and cost-effectiveness of new protocols for emergency ambulance paramedics to assess older people following a fall with referral to community-based care when appropriate. Health technology assessment. 2017;21(13):1-218.

9. Ebben RH, Vloet LC, Speijers RF, Tönjes NW, Loef J, Pelgrim T, Hoogeveen M, Berben SA. A patientsafety and professional perspective on non-conveyance in ambulance care: a systematic review. Scand J Trauma Resusc Emerg Med. 2017 Dec;25(1):71.

10. Snooks HA, Kearsley N, Dale J, Halter M, Redhead J, Foster J. Gaps between policy, protocols and practice: a qualitative study of the views and practice of emergency ambulance staff concerning the care of patients with non-urgent needs. Qual Saf Health Care. 2005;14(4):251-7.

11. Sullivan-Bolyai S, Bova C, Harper D. Developing and refining interventions in persons with health disparities: The use of qualitative description. Nurs Outlook. 2005;53(3):127-33.

12. Campbell NC, Murray E, Darbyshire J, Emery J, Farmer A, Griffiths F, et al. Designing and evaluating complex interventions to improve health care. BMJ. 2007;334(7591):455-9.

13. Corbin J, Strauss A. Basics of Qualitative Research: Techniques and Procedures for Developing Grounded Theory. 4th ed. Thousand Oaks: Sage; 2015.

14. Biernacki P, Waldorf D. Snowball Sampling: Problems and Techniques of Chain Referral Sampling. Sociol Methods Res. 1981;10(2):141-63.

15. Méabh K, Robert F. Contrasting classic, straussian, and constructivist grounded theory: Methodological and philosophical. Qual Rep. 2015;20(8):1270-89.

16. Pattenden J, Watt I, Lewin RJP, Stanford N. Decision making processes in people with symptoms of acute myocardial infarction: qualitative study. BMJ. 2002;324(7344):1006-9. 
17. Kaur R, Lopez V, Thompson DR. Factors influencing Hong Kong Chinese patients' decision-making in seeking early treatment for acute myocardial infarction. Res Nurs Heal. 2006;29(6):636-46.

18. Ahl C, Nyström M, Jansson L. Making up one's mind: - Patients' experiences of calling an ambulance. Accid Emerg Nurs. 2006;14(1):11-9.

19. King-Shier KM, Singh S, Leblanc P, Mather CM, Humphrey R, Quan H, et al. The influence of ethnicity and gender on navigating an acute coronary syndrome event. Eur $\mathrm{J}$ Cardiovasc Nurs. 2015;14(3):240-7.

20. Ridsdale L, Virdi C, Noble A, Morgan M. Explanations given by people with epilepsy for using emergency medical services: A qualitative study. Epilepsy Behav. 2012;25(4):529-33.

21. Berkhof M, van Rijssen HJ, Schellart AJ, Anema JR, van der Beek AJ. Effective training strategies for teaching communication skills to physicians: an overview of systematic reviews. Patient Educ Couns. 2011;84(2):152-62.

22. Levinson W, Lesser CS, Epstein RM. Developing physician communication skills for patient-centered care. Health Aff. 2010;29(7):1310-8.

23. Włoszczak-Szubzda A, Jarosz M, Goniewicz M. Professional communication competences perspectives of paramedics - practical and educational Anna. Ann Agric Environ Med. 2013;20(2):239-44.

24. Williams B, Beovich B, Flemming G, Donovan G, Patrick I. Exploration of difficult conversations among Australian paramedics. Nurs Health Sci. 2017;19(3):358-65.

25. Von Wyl T, Zuercher M, Amsler F, Walter B, Ummenhofer W. Technical and non-technical skills can be reliably assessed during paramedic simulation training. Acta Anaesthesiol Scand. 2009;53(1):121-7.

26. Verma SK, Willetts JL, Corns HL, Marucci-Wellman HR, Lombardi DA, Courtney TK. Falls and FallRelated Injuries among Community-Dwelling Adults in the United States. Haddad JM, editor. PLoS One. 2016;11(3):e0150939.

27. Tong A, Sainsbury P, Craig J. Consolidated criteria for reporting qualitative research (COREQ): A 32item checklist for interviews and focus groups. Int J Qual Heal Care. 2007;19(6):349-57.

\section{Figures}




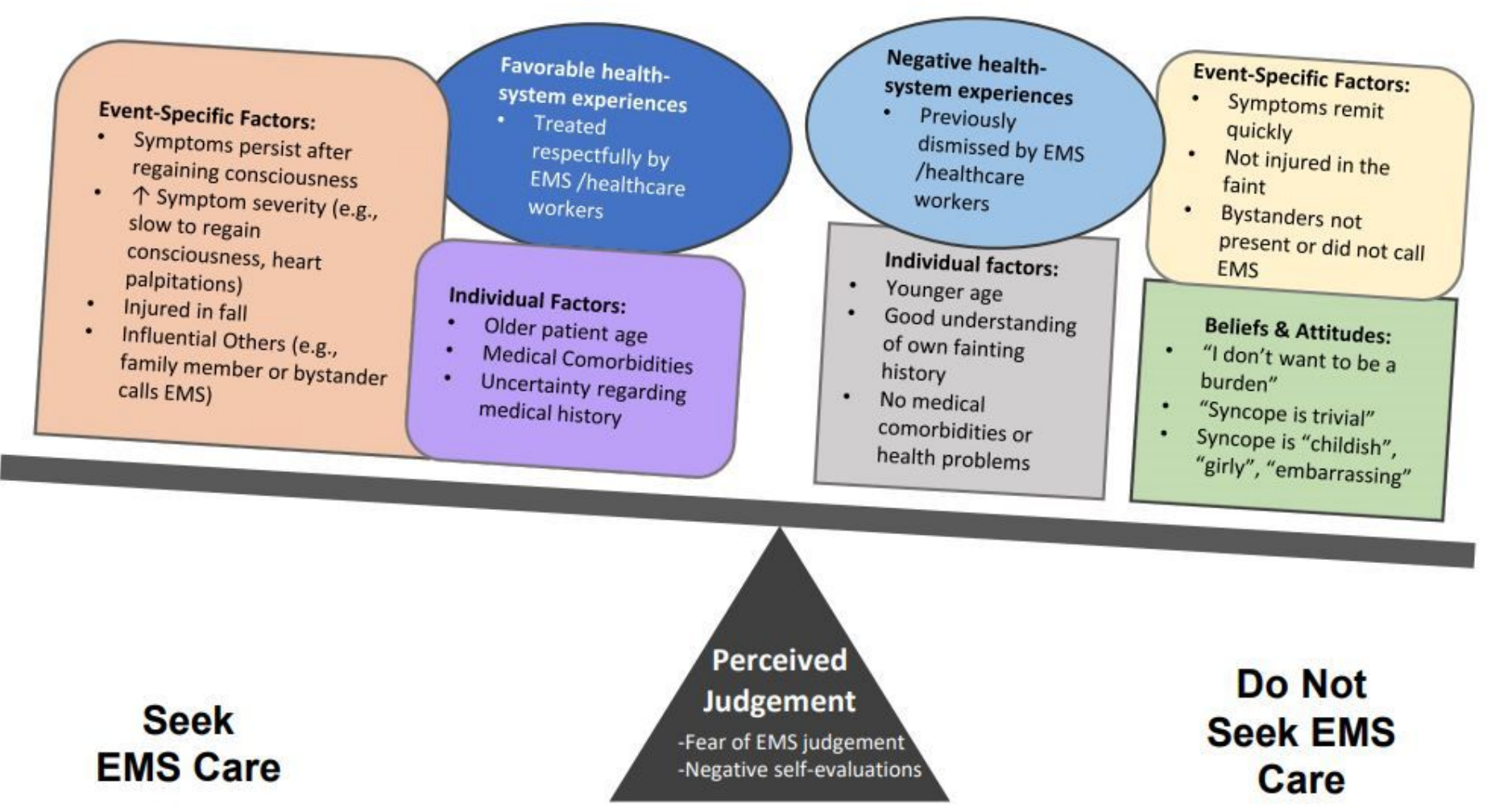

\section{Figure 1}

Factors influencing the decision to seek (or not) emergency medical assistance after fainting. Factors promoting patients' decisions not to seek EMS care frequently outweigh considerations in favor of contacting EMS. Perceived judgement underpinned the decision-making process. EMS = Emergency Medical Services. 